Año 13.

Núm. 34 Edición Especial

Revista de Investigación

Académica sin Frontera

https://revistainvestigacionacademicasinfrontera.unison.mx/index.php/RDIASF

Recibido el 23 de abril de 2020. Dictaminado mediante arbitraje favorablemente 10 de julio de 2020.

\title{
Percepción de seguridad frente a la COVID-19
}

José Marcos Bustos-Aguayo ${ }^{1}$, Cruz García-Lirios², Margarita Juárez-Nájera³

\section{Resumen}

La seguridad ha preocupado a las autoridades y a la sociedad civil durante los últimos años. Los diferentes sustratos de la sociedad tienen diferentes percepciones sobre la seguridad. El presente documento explora la confiabilidad y validez de un instrumento que mide la percepción de seguridad en los estudiantes de licenciatura al revisar siete dimensiones: territorial, nacional, público (gobierno), humano, público (autoprotección), privado e internacional. En consecuencia, establecer valores y residuos permitieron aceptar la hipótesis nula relación significativa entre las dimensiones teóricas con respecto a los factores ponderados.

Palabras clave-Fiabilidad del instrumento, validez de los instrumentos, percepción de seguridad.

\section{Security perception against COVID-19}

\begin{abstract}
Security has worried authorities and civil society in recent years. Different substrates of society have different perceptions of security. This document explores the reliability and validity of an instrument that measures the perception of security in

\footnotetext{
${ }^{1}$ PhD. Research, Department Psychology, Mexico University: marcos.bustos@unam.mx https://orcid.org/0000-0003-3423-596X

2 PhD. Research, Department Psychology, Mexico University: cgarcial213@profesor.uaemex.mx https://orcid.org/0000-0002-9364-6796

3 PhD. Research, Department Engineering, Metropolitan University: mjn@azc.uam.mx https://orcid.org/0000-0002-7447-8213
} 
Año 13.

Núm. 34 Edición Especial https://revistainvestigacionacademicasinfrontera.unison.mx/index.php/RDIASF
Revista de Investigación Académica sin Frontera ISSN: 2007-8870

Recibido el 23 de abril de 2020. Dictaminado mediante arbitraje favorablemente 10 de julio de 2020 .

undergraduate students by reviewing seven dimensions: territorial, national, public (government), human, public (self-protection), private and international. Consequently, establishing values and residuals allowed accepting the null hypothesis, a significant relationship between the theoretical dimensions with respect to the weighted factors.

Keywords -Insurance of the instrument, validity of the instruments, perception of safety.

\section{Introducción}

Al momento de escribir este artículo, la pandemia causada por el coronavirus SARS-COV-2 y la enfermedad COVID-19 ha infectado a 10 millones, ha enfermado a 5 millones y ha matado a 500,000 personas en todo el mundo. En México, ha infectado a 200,000 personas, enfermó a 150,000 y mató a 25,000 personas. En este escenario, la percepción de seguridad es un tema central en la agenda de los ciudadanos no solo debido a la crisis de salud, sino también a la crisis económica.

De esta manera, la escalada de violencia contra grupos vulnerables como niños, mujeres y ancianos ha aumentado exponencialmente y se ha sumado a los riesgos planteados por la pandemia. Derivado de esta situación, la percepción de seguridad emerge, se desarrolla y consolida como un tema central en la agenda de los ciudadanos. Es un fenómeno en el que las víctimas potenciales aprecian la pandemia como impredecible en sus efectos, inconmensurable en sus consecuencias e incontrolable por las autoridades.

Tales fenómenos, la pandemia y la seguridad, convergen en la violencia contra los grupos vulnerables como resultado de la frustración de los jefes de familia ante el desempleo, la 
Año 13.

Núm. 34 Edición Especial https://revistainvestigacionacademicasinfrontera.unison.mx/index.php/RDIASF
Revista de Investigación Académica sin Frontera ISSN: 2007-8870

Recibido el 23 de abril de 2020. Dictaminado mediante arbitraje favorablemente 10 de julio de 2020 .

hambruna y la insalubridad. De esta manera, las áreas de mayor riesgo son las más densamente pobladas, como la Ciudad de México, principalmente en la alcaldía de Iztapalapa.

Precisamente, el objetivo del presente trabajo es especificar un modelo para el estudio de la seguridad percibida frente a la pandemia, el confinamiento y la violencia hacia grupos vulnerables como los ancianos, las mujeres y los niños con respecto al jefe de familia, autoridades civiles y sanitarias.

Las contribuciones del estudio a la disciplina son 1) revisión sistemática del estado del arte, 2) sistematización de hallazgos, 3) propuesta de un modelo teórico y conceptual, 4) enfoque metodológico, 5) diagnóstico del problema, 6) discusión entre los hallazgos y la literatura revisada, 7) diseño de secuencias pedagógicas.

Por lo tanto, la primera sección revisa los enfoques teóricos y conceptuales que explican el fenómeno. La segunda sección presenta los resultados de los estudios relacionados con el tema. En la tercera sección se proponen los ejes, trayectorias y relaciones entre las variables. En la cuarta sección, se presentan las decisiones para abordar el problema. En la quinta sección, se describen los resultados. En la sexta sección, se discuten estos hallazgos. La séptima sección reflexiona sobre la contribución y la aplicación del estudio en el aula.

\section{Teoría de la percepción de seguridad.}

La seguridad, en varios países, ha sufrido una falta o ausencia, particularmente cuando se habla de participación gubernamental. La seguridad pública puede entenderse como el 
Año 13.

Núm. 34 Edición Especial https://revistainvestigacionacademicasinfrontera.unison.mx/index.php/RDIASF
Revista de Investigación Académica sin Frontera ISSN: 2007-8870

Recibido el 23 de abril de 2020. Dictaminado mediante arbitraje favorablemente 10 de julio de 2020 .

trabajo estatal para proteger y proteger a su población de los peligros o amenazas internos. En los países latinoamericanos, la seguridad pública se percibe como ausente, debido a la gran cantidad de coberturas de prensa que expone la falta mencionada (Rincón, Juárez y García, 2018).

En el caso de México, día a día, aparecen en las noticias, una mayor cantidad de coberturas de notas rojas, lo que muestra una cara violenta del país. T se estructura de la percepción de la seguridad en: Seguridad territorial; Seguridad nacional; Seguridad pública (Estado como fiscal general); Seguridad humana; Seguridad pública (autoprotección); Seguridad privada; y, percepción internauta de seguridad, alcances (Bustos, Ganga, Llamas y Juárez, 2018).

Los eventos de seguridad pública ocurren en todo el mundo y representan una amenaza para la seguridad personal, la propiedad y la defensa nacional. Los problemas de seguridad de México son como el contexto general en América Latina en muchos sentidos. Sin embargo, México tiene una influencia del crimen organizado debido a los niveles de consumo de productos ilegales en el mercado estadounidense. UU (Carreón, Blanes y García, 2018).

La seguridad pública se ha entendido tradicionalmente como la función del Estado que consiste en proteger a sus ciudadanos de ataques ilegales (o delitos contra) sus propiedades, integridad física, libertad sexual, etc. El significado de seguridad pública se infiere como la seguridad de las personas: inherencia, inseparabilidad, amplitud y enfoque en la justicia (Aldana, Rosas y García, 2018).

Se afirma que la percepción de nuestra realidad es subjetiva y que la percepción de nuestro mundo depende de nuestras condiciones de vida. La percepción de la realidad opera desde 


\section{Año 13.}

Revista de Investigación

Núm. 34 Edición Especial

Académica sin Frontera

https://revistainvestigacionacademicasinfrontera.unison.mx/index.php/RDIASF

Recibido el 23 de abril de 2020. Dictaminado mediante arbitraje favorablemente 10 de julio de 2020 .

un orden superior, desde un mesosistema que incluiría ambos (percepción y realidad), y en el que cada uno aparece como elementos y no como unidades cerradas e independientes. La noción de que: lo que vemos, podría no ser lo que realmente existe, ha perturbado y atormentado a toda la población en cada sector, clase o rol de nuestra sociedad. Los diferentes sectores de la población tendrían una percepción diferente de la seguridad (Carreón, García y Blanes, 2018).

También se puede mencionar que el estigma cultural en el país también afecta y promueve la falta de custodia pública, debido a la forma general de pensar de la población mexicana, que, en comparación con otras culturas, parece ser lenta y con poco interés. para desarrollar en aspectos académicos, profesionales, sociales, entre otros. La administración de la seguridad pública es la implementación de políticas públicas que justifican la orientación del Estado en la prevención del delito y la administración de justicia, pero solo la desconfianza de los ciudadanos hacia la acción del gobierno se evidencia por una creciente percepción de inseguridad reportada en la literatura. en siete dimensiones: territorial, nacional, público (gobierno), humano, público (autoprotección), privado e internacional (Martínez, Anguiano y García, 2018).

México se puede ver desde diversos ámbitos como económico, histórico o social. En ese sentido, existen otros sub-ámbitos (o sub-escalas en el ámbito social) como salud, seguridad pública, educación, conciencia ambiental, entre otros. Como se mencionó anteriormente, los diferentes sectores de la población tienen una percepción diferente de los sub-ámbitos sociales (o sub-escalas). En el caso de los estudiantes de licenciatura, ya que su formación académica da la oportunidad de generar una forma crítica de pensar, el sector de la población 
Año 13.

Núm. 34 Edición Especial https://revistainvestigacionacademicasinfrontera.unison.mx/index.php/RDIASF
Revista de Investigación Académica sin Frontera ISSN: 2007-8870

Recibido el 23 de abril de 2020. Dictaminado mediante arbitraje favorablemente 10 de julio de 2020.

puede generar una percepción sólida del factor que afecta el contexto de la sociedad (García, Carreón y Hernández, 2017).

\section{Estudios de percepción de seguridad.}

La teoría de la percepción de seguridad alude a dimensiones que son convergentes con respecto a la confianza entre gobernantes y gobernados. De esta manera, la premisa central de la teoría es que los ciudadanos tienen expectativas desfavorables o positivas de sus autoridades a cargo de la aplicación de la ley y la prevención del delito, así como la rehabilitación social (Carreón, 2020). En este sentido, la seguridad es un fenómeno sociopolítico, pero se reduce a las expectativas de los medios sobre la acción del gobierno, así como a la desconfianza o empatía por sus estrategias, programas o políticas en términos de salvaguardar la integridad y la dignidad de sus gobernados, así como privados propiedad e intereses públicos.

Las dimensiones de esta percepción de seguridad se han estructurado en términos socioespaciales como el territorio o en cuestiones sociales como la nación, pero con énfasis en la situación de sectores, estratos o grupos como la denominada seguridad pública y ciudadana. , así como los intereses individuales como la seguridad privada e Internet (García, 2019) .

La seguridad territorial y nacional ha sido tradicionalmente la más abordada desde la sociología del riesgo para explicar el impacto del cambio climático en el nivel del mar y las costas, así como los eventos de riesgo derivados de sequías, heladas, incendios, inundaciones 


\section{Año 13.}

Revista de Investigación

Núm. 34 Edición Especial

Académica sin Frontera

https://revistainvestigacionacademicasinfrontera.unison.mx/index.php/RDIASF

Recibido el 23 de abril de 2020. Dictaminado mediante arbitraje favorablemente 10 de julio de 2020.

o terremotos en áreas vulnerables, el tráfico de especies o la aparición de epidemias debido a la invasión de territorios animales (Quiroz, 2019).

La llamada bioseguridad se enfoca en los alimentos como el principal indicador del nivel de salud ante una crisis de salud o ambiental (Hernández, 2019). En este sentido, la seguridad territorial o nacional debería haberse especializado en público porque cada sector o estrato social demandaba diferentes necesidades según las situaciones contingentes. La ciudadanía de esta seguridad dio paso a la individualización de las expectativas y los recursos, lo que condujo a la autoprotección personal y virtual, con el surgimiento de la ciberseguridad.

Existen más diferencias entre la bioseguridad y la ciberseguridad, pero ambas son esenciales para el estado de derecho, la administración de justicia, la adquisición del delito, la rehabilitación social y la pacificación colectiva. Desde una perspectiva tradicional, ambas dimensiones son observables como complementarias, pero desde un enfoque progresivo se asumen como concomitantes. En otras palabras, los efectos del cambio climático son cada vez más vinculadas al robo de identidad, extorsión o C o cooptación, ya que los nichos de deterioro ambiental y social que se originan los delitos informáticos se asumen. $\mathrm{O}$, en base a la ciberseguridad, los datos de robos, secuestros $\mathrm{u}$ homicidios en situaciones de desastres naturales o contingencias de salud se observan en tiempo real.

\section{Especificación de un modelo para el estudio de la percepción de seguridad.}

A partir de la revisión teórica, conceptual y empírica, se modelaron las relaciones entre las variables. De esta manera, la seguridad territorial y nacional son concomitantes dado su nivel de generalidad en la protección del país, así como el multilateralismo involucrado en medidas 


\section{Año 13.}

Revista de Investigación

Núm. 34 Edición Especial

Académica sin Frontera

https://revistainvestigacionacademicasinfrontera.unison.mx/index.php/RDIASF

Recibido el 23 de abril de 2020. Dictaminado mediante arbitraje favorablemente 10 de julio de 2020.

de pacificación internacionales o regionales. En los casos de seguridad pública y ciudadana, ambos comparten los imperativos de salvaguardar los bienes comunes que, aunque son públicos, pueden establecerse como disponibles social y ambientalmente para las generaciones futuras. En este sentido, la seguridad privada y digital también es similar en términos de prevención de delitos que amenazan la dignidad e integridad del individuo en lugar de la sociedad.

Hipótesis nula: las relaciones teóricas entre las percepciones de seguridad son consistentes con los datos observados. Esto es así porque se presume que el instrumento mide las siete dimensiones de seguridad, así como su consistencia cuando se aplica a toros, escenarios y muestras. Además, la seguridad como fenómeno multidimensional sugiere niveles de medición concomitantes (HC) entre sí, reflexivos (HR) y con errores (HE) atribuidos a la variación de las respuestas.

Las hipótesis concomitantes aluden a las covarianzas entre las dimensiones del fenómeno, así como a la explicación de su estructura de trayectoria si surgiera una nueva especificación o modelado al probar la hipótesis nula (García, 2020).

Las hipótesis reflectantes aluden a las relaciones entre los factores con respecto a los indicadores, lo que sugiere la estructuración del fenómeno, así como la convergencia de las respuestas a los reactivos que miden cada característica de las dimensiones (Rivera, 2020).

Las hipótesis de errores de medición se refieren a variaciones inexplicables en la estimación de la estructura de las relaciones concomitantes y reflexivas. Además, sugiere la probable incidencia de otros factores e indicadores no incluidos en el modelo (Amemiya, 2020). 
Año 13.

Núm. 34 Edición Especial https://revistainvestigacionacademicasinfrontera.unison.mx/index.php/RDIASF
Revista de Investigación Académica sin Frontera ISSN: 2007-8870

Recibido el 23 de abril de 2020. Dictaminado mediante arbitraje favorablemente 10 de julio de 2020 .

\section{Método}

Diseño. Estudio no experimental, exploratorio y transversal. La muestra se realizó con una selección no aleatoria de 320 estudiantes $\left(\mathrm{M}=21.3 \mathrm{SD}=2.1\right.$ edad y $\mathrm{M}=9^{\prime} 865.23 \mathrm{SD}=$ 345.32 ingreso mensual) en una universidad pública en el Estado de México. El 44\% son mujeres y el $66 \%$ son hombres.

El 50\% de las personas encuestadas son menores de 18 años $(M=17.29$ y $S D=1.24)$, el $40 \%$ tiene entre 18 y 22 años $(M=20.14$ y $S D=2.36)$ y el $10 \%$ más de 22 años $(M=23.25$ y $S D=4.36)$ El $30 \%$ admitió menos de 3'500 pesos $(M=3 ' 200$ y $S D=123.25)$, el $25 \%$ entre 3'500 y 7'000 mensualmente $\left(M=5^{\prime} 467\right.$ y $\left.S D=345.25\right)$ y el $45 \%$ ingresa más de 7 '000 mensuales $(M=8 ' 913$ y $S D=135.47)$.

Instrumento. Se construyó una percepción de escala de la inseguridad que incluye la percepción de 280 reactivos en torno a la seguridad: territorial, nacional, pública (gobierno), humana, pública (autoprotección), privada e internauta.

Percepción de subescala de seguridad territorial. Se refiere a las expectativas sobre el estado como rector de la paz pública (Mendoza, Carreón, Mejía y García, 2017). Incluye cuatro reactivos que responden con cualquiera de las seis opciones de respuesta: $0=$ "nada probable" a 5 = "muy probable".

Percepción de la subescala de la seguridad nacional. Se refiere a las expectativas sobre el Estado como procurador de delitos contra la democracia, la identidad nacional o los intereses de la población (García, Carreón y Hernández, 2017). Incluye cuatro reactivos que responden con cualquiera de las seis opciones de respuesta: $0=$ "nada probable" a 5 = "muy probable". 


\section{Año 13.}

Revista de Investigación

Núm. 34 Edición Especial

Académica sin Frontera

https://revistainvestigacionacademicasinfrontera.unison.mx/index.php/RDIASF

Recibido el 23 de abril de 2020. Dictaminado mediante arbitraje favorablemente 10 de julio de 2020 .

Percepción de subescala de seguridad pública. Expectativas con respecto al alusivo del estado como fiscal general (Juárez, Carreón, Quintero, Espinoza, Bustos y García, 2017). Incluye cuatro reactivos que se responden con cualquiera de las seis opciones de respuesta: 0 = "nada probable" a 5 = "muy probable".

Percepción de la subescala de la seguridad humana. Se refiere a las expectativas generadas ante las políticas de prevención del delito, la administración de justicia y la promoción de la paz social (Quintero, Hernández, Sánchez, Molina y García, 2017). Incluye cuatro reactivos que responden con cualquiera de las seis opciones de respuesta: $0=$ "nada probable" a $5=$ "muy probable".

Percepción de la subescala de la seguridad pública. Se refiere a las expectativas que los ciudadanos generaron a partir de la desconfianza y alienación del Estado con sus instituciones de seguridad, mientras que el interés se centra en los recursos civiles para la autoprotección (Carreón, Hernández y García, 2017). Incluye cuatro reactivos que responden con cualquiera de las seis opciones de respuesta: $0=$ "nada probable" a 5 = "muy probable".

Percepción de la subescala de la seguridad privada. Se refiere a las expectativas de la sociedad civil generadas por el Estado como incapaces de prevenir el crimen y combatir la corrupción (García, Carreón y Hernández, 2016). Incluye cuatro reactivos que se responden con cualquiera de las seis opciones de respuesta: $0=$ "nada probable" a 5 = "muy probable".

Subescala Percepción internauta de seguridad. Se refiere a las expectativas que los usuarios de Internet consideran generadas por el espía estatal en su búsqueda de información, selección de contenido y difusión de temas (Mejía, Carreón y García, 2016). Incluye cuatro 
Año 13.

Núm. 34 Edición Especial https://revistainvestigacionacademicasinfrontera.unison.mx/index.php/RDIASF
Revista de Investigación Académica sin Frontera ISSN: 2007-8870

Recibido el 23 de abril de 2020. Dictaminado mediante arbitraje favorablemente 10 de julio de 2020 .

reactivos que se responden con cualquiera de las seis opciones de respuesta; $0=$ "nada probable" a 5 = "muy probable".

Se utilizó la técnica Delphi para establecer la homogeneidad de los conceptos en los reactivos. Examinamos la exposición en el vestíbulo de la biblioteca de su universidad. Los datos se procesaron con el Paquete de Análisis Estadístico para Ciencias Sociales (SPSS por sus siglas en inglés) y el Análisis Estructural de Momentos (AMOS por sus siglas en inglés). 18,0 versiones. Se estimó la confiabilidad con el alfa de Cronbach, la prueba de validez de Bartlett, KMO y el peso factorial.

El alfa de Cronbach se estimó para establecer la consistencia interna de la escala general y las subescalas. El parámetro Bootstrap se calculó para establecer el muestreo cuando no es posible utilizar completamente los datos y solo se utiliza una parte de la distribución. Se calculó la adecuación y la esfericidad con los parámetros de Kayser Meyer Olkin y la prueba de Bartlett. Se realizó un análisis factorial exploratorio con rotación de promax de ejes principales y criterio de oblicuidad. La prueba de la hipótesis se realizó con la estimación de los parámetros de ajuste y residual.

Se realizó un segundo estudio con 89 comerciantes de una localidad con un alto índice de inseguridad pública, considerando su participación en la prevención del delito y el empleo de la seguridad privada. Se utilizó la misma escala y la información se procesó con el mismo software y parámetros.

\section{Resultados}


Año 13.

Núm. 34 Edición Especial https://revistainvestigacionacademicasinfrontera.unison.mx/index.php/RDIASF
Revista de Investigación

Académica sin Frontera

ISSN: 2007-8870

Recibido el 23 de abril de 2020. Dictaminado mediante arbitraje favorablemente 10 de julio de 2020 .

Consistencia interna, de acuerdo con la recolección y análisis de datos, en la escala general (0.793) y las subescalas F1 = Percepción de seguridad territorial (alfa de 0.792 y $22 \%$ de la varianza total explicada); F2 = Percepción sobre seguridad nacional (alfa de 0.709 y $19 \%$ de la varianza explicada); F3 = Percepción de seguridad pública (alfa de 0.785 y 17\% de la varianza explicada); F4 = Percepción de la seguridad humana (alfa de 0.782 y 14\% de la varianza explicada); F5 = Percepción de seguridad pública (alfa de 0.792 y $12 \%$ de la varianza explicada); F6 = Percepción de seguridad privada (alfa de 0.794 y 9\% de la varianza explicada); y F7 = La percepción de la seguridad interna (alfa de 0.731 y $7 \%$ de la varianza explicada) es discreta porque evidencia las diferencias entre los contextos de estudio en los que se conceptualizó la seguridad.

Tabla 1. Descriptivo del instrumento

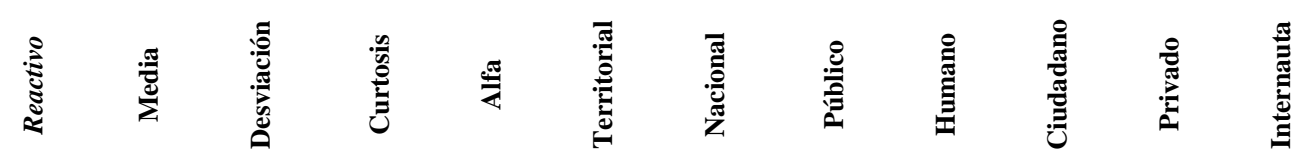

\begin{tabular}{|c|c|c|c|c|c|c|c|c|}
\hline$r 1$ & 1.3 & 59 & 1,2 & .79 & & & & 37 \\
\hline$r 2$ & 1,3 & 51 & 1,4 & .70 & & & & 31 \\
\hline$r 3$ & 1,4 & .36 & 1,5 & .79 & & & & .36 \\
\hline$r 4$ & 1,7 & 93 & 1,6 & .77 & & & & .38 \\
\hline$r 5$ & 1,7 & 83 & 1,9 & .78 & & & .47 & \\
\hline$r 6$ & 1,1 & 53 & 1,8 & .75 & & & .46 & \\
\hline$r 7$ & 1.4 & 47 & 1,6 & .71 & & & .40 & \\
\hline$r 8$ & 1,3 & , 43 & 1,7 & .78 & & & .41 & \\
\hline$r 9$ & 3.4 & 88 & 1,2 & 71 & & .30 & & \\
\hline$r 10$ & 3.7 & 69 & 1.1 & .73 & & .36 & & \\
\hline$r 11$ & 3.4 & 91 & 1,5 & .78 & & .34 & & \\
\hline$r 12$ & 3,1 & .50 & 1,3 & .70 & & 31 & & \\
\hline$r 13$ & 1,4 & .50 & 1,0 & .77 & .30 & & & \\
\hline$r 14$ & 1,8 & 63 & 1,1 & .73 & .30 & & & \\
\hline
\end{tabular}


Año 13. Académica sin Frontera

Núm. 34 Edición Especial ISSN: 2007-8870 https://revistainvestigacionacademicasinfrontera.unison.mx/index.php/RDIASF

Recibido el 23 de abril de 2020. Dictaminado mediante arbitraje favorablemente 10 de julio de 2020.

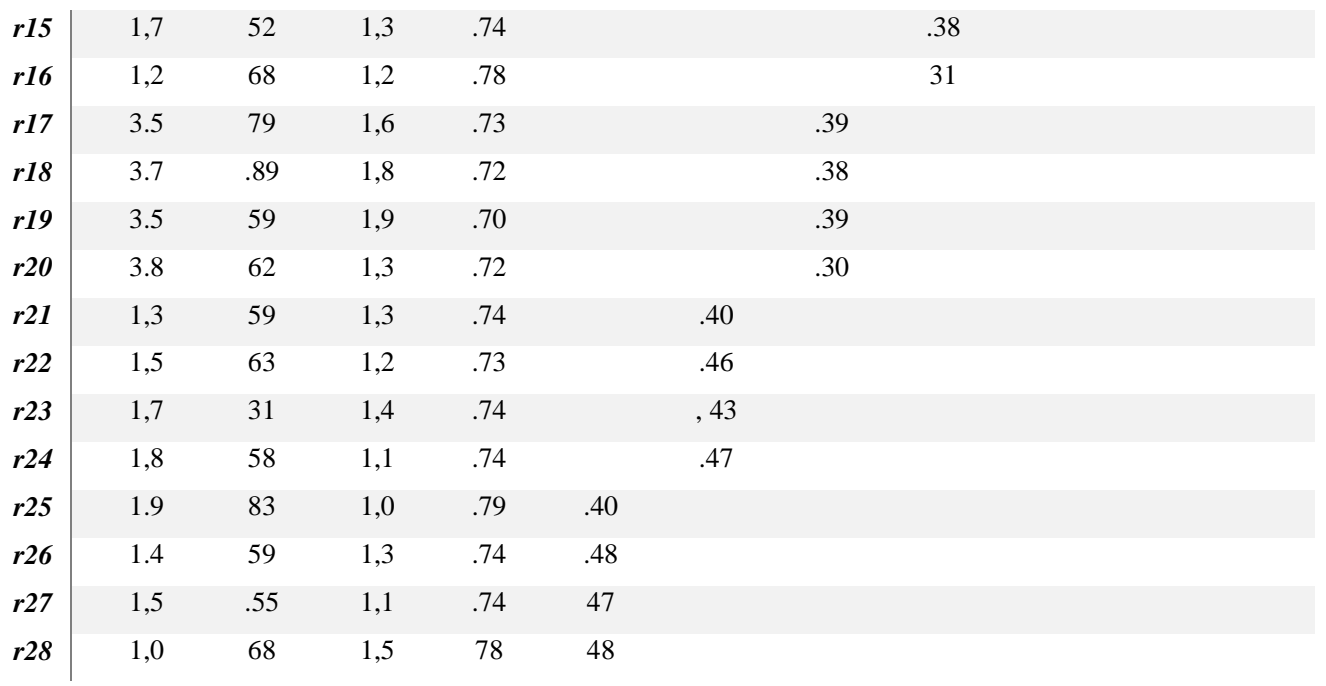

Nota: Elaborado con estudio de datos; $R=$ Reactivo, $M=$ Medios, $S=$ Desviación estándar, $K=$ Kurtosis, $A$ $=$ Alfa de Cronbach. Adecuación (KMO =, 764), Esfericidad $\left\lceil\chi^{2}=334,1\right.$ (25gl) $\left.p<, 01\right\rceil$ Método: Formas principales, Rotación: Promax. Percepción de seguridad territorial (alfa de 0.792 y $17 \%$ de la varianza total explicada); Percepción sobre seguridad nacional (alfa de 0,709 y $15 \%$ de la varianza explicada); Percepción de seguridad pública (alfa de 0.785 y 11 \% de la varianza explicada); Percepción de la seguridad humana (alfa de 0.782 y8\% de la varianza explicada); Percepción de la seguridad ciudadana (alfa de 0.792 y $5 \%$ de la varianza explicada); Percepción de seguridad privada (alfa de 0.794 y 3 \% de la varianza explicada); y Percepción de seguridad internauta (alfa de 0.731 y 1 \% de la varianza explicada).

Un segundo estudio, una vez que se establecieron los factores, se estimaron las posibles relaciones lineales asociativas para investigar la emergencia de un factor de segundo orden común a los siete factores de primer orden encontrados (ver Tabla 2).

Tabla 2. Correlaciones y covariaciones de los factores. 
Año 13.

Núm. 34 Edición Especial

Revista de Investigación

Académica sin Frontera

ISSN: 2007-8870

https://revistainvestigacionacademicasinfrontera.unison.mx/index.php/RDIASF

Recibido el 23 de abril de 2020. Dictaminado mediante arbitraje favorablemente 10 de julio de 2020.

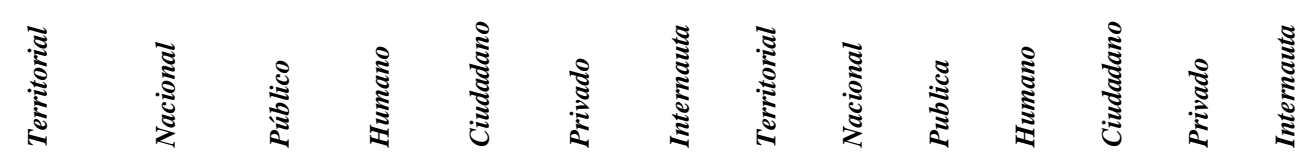

\begin{tabular}{|c|c|c|c|c|c|c|c|c|c|c|c|c|c|c|}
\hline Territorial & 1,0 & & & & & & & 1,9 & & & & & & \\
\hline Nacional &, $32 * * *$ & 1,0 & & & & & & 45 & 1,8 & & & & & \\
\hline Público & $31 *$ & $31 *$ & 1,0 & & & & & 41 & 32 & 1,7 & & & & \\
\hline Humano &, $35 * *$ &, $32 *$ &, $30 *$ & 1,0 & & & & 48 & 49 &, 43 & 1,6 & & & \\
\hline Ciudadano &, $39 *$ &, $33 * *$ & $36 *$ & $36 * *$ & 1,0 & & & 40 & 40 & 41 & 40 & 1,8 & & \\
\hline Privado & $34 *$ &, $32 *$ & $38 *$ & $34 *$ &, $39 *$ & 1,0 & &, 43 & 41 & 37 & 47 &, 43 & 1,5 & \\
\hline Internauta &, $30 *$ &, $31 * *$ & $31 *$ &, $35 *$ &, $32 *$ & $36 * *$ & 1,0 & 47 &, 43 &, 43 & 45 & 42 & 41 & 1,9 \\
\hline
\end{tabular}

Nota: Elaborado con estudio de datos; Percepción de la seguridad territorial; P erception en la seguridad nacional; Percepción de seguridad pública; Percepción de la seguridad humana; Perception de Citizen seguridad; Percepción de seguridad privada; Percepción de seguridad internauta: ${ }^{*} p<, 01$; *** $p$ $<, 001 ; * * *<<, 0001$

Una vez que se establecieron las relaciones entre los factores, sus trayectorias se modelaron con respecto a un factor común que la literatura identifica como una percepción de seguridad para explicar un fenómeno emergente de conciencia, prevención y demanda del estado de derecho con la administración de justicia.

Las relaciones entre factores, perturbaciones, indicadores y errores se especificaron para probar las hipótesis convergentes (HC) entre los factores de primer orden con respecto a un factor común de segundo orden, las hipótesis de perturbación (HD) que explican el influencia de otros factores en el modelo, las hipótesis reflexivas (HR) que establecen los factores latentes de primer orden a partir de sus indicadores y las hipótesis de error (HE) que explican la incidencia de otros indicadores en los factores de primer orden (Sandoval, 2020) . 


\section{Año 13.}

Revista de Investigación

Núm. 34 Edición Especial

Académica sin Frontera

https://revistainvestigacionacademicasinfrontera.unison.mx/index.php/RDIASF

Recibido el 23 de abril de 2020. Dictaminado mediante arbitraje favorablemente 10 de julio de 2020 .

Los valores de los parámetros de ajuste y residuales $\lceil\chi 2=135.34(32 \mathrm{gl}) \mathrm{p}=0.054$; GFI $=$ 0,995; CFI = 0.990; RMSEA = 0,003 sugiere el no rechazo de la hipótesis nula en relación con las diferencias significativas entre las relaciones teóricas establecidas en la literatura con respecto a las relaciones empíricas encontradas en el estudio.

\section{Discusión}

El objetivo del presente trabajo fue confirmar la estructura factorial de la percepción de seguridad, aunque el diseño de la investigación limita los hallazgos a la muestra de investigación, sugiriendo la extensión del trabajo hacia la expansión de los factores, ya que en su totalidad explican $55 \%$

En relación con la teoría de la percepción de seguridad, que plantea nueve dimensiones relacionadas con territorio, nación, ciudadanía, público, privado, humano e internet, este trabajo demostró que la seguridad humana es el factor que más refleja la estructura de seguridad perceptiva. Las líneas de estudio que se refieren a las dimensiones de la seguridad humana nos permitirán notar escenarios de conflicto entre gobernantes y gobernados, así como el surgimiento de la seguridad ciudadana y privada.

Con respecto a los estudios de seguridad donde se destaca un estado continuo coercitivo y persuasivo en su relación con los ciudadanos, este trabajo ha demostrado que la seguridad humana es una dimensión que explica las diferencias y similitudes entre gobernantes y gobernados. El desarrollo de esta dimensión nos permitirá notar la transición de un sistema coercitivo a otro persuasivo. Es decir, la seguridad atribuida a la violencia legítima de un gobierno democrático se observará hasta la seguridad que separa al régimen de toda 
Año 13.

Núm. 34 Edición Especial https://revistainvestigacionacademicasinfrontera.unison.mx/index.php/RDIASF
Revista de Investigación Académica sin Frontera ISSN: 2007-8870

Recibido el 23 de abril de 2020. Dictaminado mediante arbitraje favorablemente 10 de julio de 2020.

responsabilidad y recarga al ciudadano con la atribución de prevención al limitar su expresión y su propiedad.

Con respecto al modelado de las siete dimensiones de la percepción de seguridad, el presente estudio ha demostrado que explican el 55\% de la varianza y advierten sobre la aparición de un factor común que la literatura identifica como de segundo orden. Las líneas de investigación sobre el surgimiento de este factor común permitirán evaluar, acreditar y certificar las relaciones entre gobernantes y gobernadores en materia de seguridad multidimensional.

En resumen, la seguridad percibida es un fenómeno psicológico multidimensional, ya que deriva de las relaciones entre las autoridades y los ciudadanos con respecto a la prevención del delito, la administración de justicia y la rehabilitación social, aunque otras dimensiones, como la seguridad sectorial o mediática, explican el impacto de las políticas. estrategias y programas sobre decisiones y acciones civiles.

\section{Conclusión}

El objetivo de este trabajo fue corroborar la estructura factorial de la seguridad percibida, aunque el diseño de la investigación limita el hallazgo del escenario de investigación, sugiere la construcción de una agenda y la incidencia en las políticas de seguridad basadas en las opiniones y expectativas de los gobernados con respecto a La actuación de sus gobernantes.

En México, prevalece una interpretación o idea común de qué país carece de seguridad. La ausencia de custodia está influenciada por la presencia del crimen organizado, la venta ilegal 
Año 13.

Núm. 34 Edición Especial https://revistainvestigacionacademicasinfrontera.unison.mx/index.php/RDIASF
Revista de Investigación Académica sin Frontera ISSN: 2007-8870

Recibido el 23 de abril de 2020. Dictaminado mediante arbitraje favorablemente 10 de julio de 2020 .

de drogas y armas, y la corrupción disponible en cada rama del gobierno, entre los aspectos principales.

Las correlaciones de fiabilidad y validez cuando la unidad muestra lejos que hay otras dimensiones vinculadas a la construcción. En este sentido, la inclusión del autocontrol explica los efectos de la propaganda estatal en materia de prevención del delito, aplicación de la ley y educación para la paz en los estilos de vida de los sectores civiles.

La contribución de este estudio se refiere a la confiabilidad y validez de un instrumento que mide siete dimensiones de seguridad: territorial, nacional, humana, pública, pública, privada y digital.

Los estudios sobre seguridad pública identifican en las expectativas del gobierno el factor predominante que explica el fenómeno como una institución eficiente, efectiva y efectiva, pero en el presente trabajo la aparición de este fenómeno se ha demostrado a partir de una estructura de percepciones en torno a lo personal, ciudadano, agenda pública, humana, nacional y territorial.

\section{Referencias}

Aldana, WI Rosas, FJ \& Garcia , C. , (2018). Especificación de un modelo para el estudio de la agenda de la seguridad pública. Atlante, 9 (1), 1-20 
Año 13.

Núm. 34 Edición Especial https://revistainvestigacionacademicasinfrontera.unison.mx/index.php/RDIASF
Revista de Investigación Académica sin Frontera ISSN: 2007-8870

Recibido el 23 de abril de 2020. Dictaminado mediante arbitraje favorablemente 10 de julio de 2020 .

Amemiya, M. (2020). Metanálisis retrospectivo del efecto aleatorio y homogéneo de la validez de la escala de percepción de riesgo. American Journal of Applied Scientific Research, 10 (4), 25-35

Bustos, JM Ganga, FA Llamas, B. y Juárez, M. (2018). Contrastación de un modelo de decisión prospectiva e implicaciones para una gobernanza universitaria de la sustentabilidad. Margen, 89 (1), 1-16

Carreon, J . (2020). Redes neuronales de escenarios, fases y discursos de violencia de Internet. Journal of Neurology \& Ne U to Toxicology, 4 (3), 1-9

Carreón, J. Blanes, AV y García, C. (2018). Confiabilidad y validez de un modelo de gobernanza percibida de la inseguridad. Sin Frontera, 11 (27), 1-53

Carreon, J. García, C. y Blanes, AV (2018). Redes de violencia en torno a la gobernanza de la seguridad pública, Ciencias Sociales, 4 (2), 60-65

Carreón, J. Hernández, J. y García C. (2017). Una revisión teórica para el estudio de la gobernanza de la seguridad pública. Epsys , 4 (1), 1-15

García, C. (2019). Estructura factorial exploratoria de la seguridad pública. Revista Internacional de Sistemas, 23 (1), 82-86

García, C. (2020). Especificación de un modelo para el estudio de sistemas de inseguridad. Global Journal of Management \& Business Research, 20 (1), 7-10 
Año 13.

Núm. 34 Edición Especial https://revistainvestigacionacademicasinfrontera.unison.mx/index.php/RDIASF
Revista de Investigación Académica sin Frontera ISSN: 2007-8870

Recibido el 23 de abril de 2020. Dictaminado mediante arbitraje favorablemente 10 de julio de 2020 .

García, C. Carreón, J. y Hernández, J, (2017). Gobernanza de la seguridad pública. Revisión de la literatura para una discusión del estado del conocimiento de la identidad sociopolítica delictiva. Margen, 84 (1), 1-17

García, C. Carreón, J. y Hernández, J. (2017). La cogestión como dispositivo de seguridad para el desarrollo sustentable local. Eureka, 14 (2), 268-289

Garcia, C., Carreón, J. y Hernández, J. (2016). Gobernanza del terror a la delincuencia. Eureka, 13 (2), 168-185

Hernández, J. (2019). Acoso por internet en la agenda documental y de expertos. Asian Journal of Science \& Technology, 10 (8), 1-3

Hernández, J. (2020). Especificación de un modelo de violencia sexual. British Journal of Medical \& Health Science, 2 (2), 1-4

Juárez, M. Carreon, J. Quintero, M. L. Espinoza, F. Bustos, J. M. y García, C. (2017). Fiabilidad y validez de un instrumento que mide la dimensión de seguridad y percepción de riesgo en estudiantes de una universidad pública. Revista Internacional de Avances en Ciencias Sociales y Humanidades, 11 (12), 23-13

Martínez, E. Anguiano, F. y García, C. (2018). Gobernanza de las obras sociales hacia una red de violencia. Social. Science Learning Educational Journal, 6 (1) , 1-3

Mejía, S. Carreón, J. y García, C. (2016). Efectos psicológicos e violencia e inseguridad en adultos mayores. Eureka, 13 (1), 39-55 
Año 13.

Núm. 34 Edición Especial https://revistainvestigacionacademicasinfrontera.unison.mx/index.php/RDIASF
Revista de Investigación Académica sin Frontera ISSN: 2007-8870

Recibido el 23 de abril de 2020. Dictaminado mediante arbitraje favorablemente 10 de julio de 2020 .

Mendoza, D. Carreón, J., Mejía, S. y García, C. (2017). Especificación de un modelo de representaciones propagandísticas en adultos mayores ante la seguridad pública. Tlatemoani, 25 (1), 21-31

Quintero, M. L Hernandez, J. Sánchez, A. Molina, HD y García, C. (2017). Modelo de expectativas en torno a la seguridad pública en microempresarios del centro de México. Sin Frontera, 10 (26), 1-20

Quiroz, C. Y. (2019). Retrospectiva metanalítica de las políticas en el manejo del riesgo de transporte en la Ciudad de México. Net Journal of Social Science, 7 (4), 92-100

Rincón, R. M. Juárez, M. y García, C. (2018). Interpretación de Discursos En torno al habitus de Movilidad para develar el significado del Transporte Público. Margen, 90 (1), 1-13

Rivera, B. L. (2020). Algoritmo estructural exploratorio del factor de riesgo percibido. Revista Internacional de Invenciones de Humanidades y Ciencias Sociales. 10 (8), 26-30

Sandoval, F. J. (2020). Expectativas laborales frente a eventos de riesgo y efectos sociales colaterales. Revista Internacional de Humanidades, Ciencias Sociales y Educación. 7 (5), $1-7$ 


\section{Año 13.}

Revista de Investigación

Núm. 34 Edición Especial Académica sin Frontera https://revistainvestigacionacademicasinfrontera.unison.mx/index.php/RDIASF

Recibido el 23 de abril de 2020. Dictaminado mediante arbitraje favorablemente 10 de julio de 2020.

\section{Directorio Institucional}

Dr. Enrique Fernando Velázquez Contreras

Rector

Dr. Ramón Enrique Robles Zepeda

Secretario General Académico

Dra. Rosa María Montesinos Cisneros

Secretaria General Administrativa

Dr. Rodolfo Basurto Álvarez

Director de Vinculación y Difusión

Dra. Adriana Leticia Navarro Verdugo

Vicerrectora de la Unidad Regional Sur

Dr. Ernesto Clark Valenzuela

Director de la División de Ciencias Económicas y Sociales

Dr. Francisco Espinoza Morales

Secretario de la División de Ciencias Económico y Sociales

Dra. Leticia María González Velásquez

Jefe del Departamento de Ciencias Económico Administrativas

Dra. Lidia Amalia Zallas Esquer

Jefe de Departamento de Ciencias Sociales 
Año 13.

Revista de Investigación

Núm. 34 Edición Especial

Académica sin Frontera

https://revistainvestigacionacademicasinfrontera.unison.mx/index.php/RDIASF

Recibido el 23 de abril de 2020. Dictaminado mediante arbitraje favorablemente 10 de julio de 2020.

\section{Comité Directivo}

\section{Editor Responsable}

Dr. Francisco Espinoza Morales

\section{Directora}

Dra. Leticia María González Velásquez

\section{Subdirector}

Dr. Javier Carreón Guillen

\section{Editor Científico}

Dr. Cruz García Lirios

\section{Master Gráfico}

M.T.I. Francisco Alan Espinoza Zallas

Nos complace anunciar que su diario, "Academic Research Journal Withoutborders" (ISSN/EISSN 2007-8870) fue evaluado positivamente en la indexación Citefactor, ahora la página de la revista está disponible en línea, en caso de cualquier problema.

Journals Master | International Innovative Journal Impact Factor (IIJIF)

Red Latinoamericana de revistas Académicas en Ciencias Sociales y Humanidades

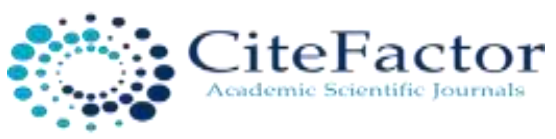

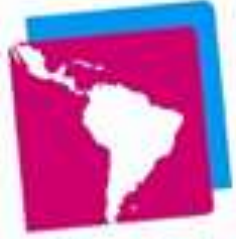

LatinREV

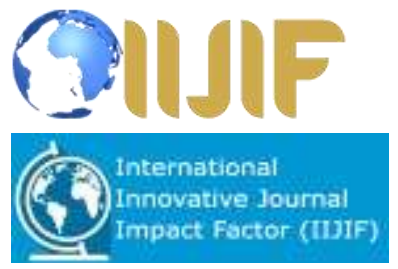




\section{Año 13.}

Revista de Investigación

Núm. 34 Edición Especial

Académica sin Frontera

https://revistainvestigacionacademicasinfrontera.unison.mx/index.php/RDIASF

Recibido el 23 de abril de 2020. Dictaminado mediante arbitraje favorablemente 10 de julio de 2020.

\section{Comité editorial}

Dra. Angélica María Rascón Larios

Universidad de Sonora. México

Dra. María del Rosario Molina González

Universidad de Sonora

Dra. Francisca Elena Rochin Wong

Universidad de Sonora. México

Dra. Lidia Amalia Zallas Esquer

Universidad de Sonora. México

Dra. Beatriz Llamas Arechiga

Universidad de Sonora. México

Dr. Rogelio Barba Álvarez

Universidad de Guadalajara. México

Dra. Rosa María Rincón Ornelas

Universidad de Sonora. México

Dr. Juan Flores Preciado

Universidad de Colima. México

Dr. Amado Olivares Leal. Universidad de Sonora

Universidad de Sonora. México

Dr. Guillermo Velázquez Valadez.

Instituto Politécnico Nacional (IPN) México

Dr. Hugo Nefstalí Padilla Torres.

Universidad Estatal de Sonora. México

Dr. Luis Ramón Moreno Moreno.

Universidad Autónoma de Baja California. México

Dr. Miguel Ángel Vázquez Ruiz.

Universidad de Sonora. México 
Año 13.

Revista de Investigación Académica sin Frontera

Núm. 34 Edición Especial ISSN: 2007-8870 https://revistainvestigacionacademicasinfrontera.unison.mx/index.php/RDIASF

Recibido el 23 de abril de 2020. Dictaminado mediante arbitraje favorablemente 10 de julio de 2020.

Dra. Lorena Vélez García.

Universidad Autónoma de Baja California. México

Dra. Pabla Peralta Miranda.

Universidad Simón Bolívar, Barranquilla, Colombia

Mtro. Roberto Espíritu Olmos

Universidad de Colima (FCA Tecomán) Colima

Dr. Héctor Priego Huertas.

Universidad de Colima (FCA Tecomán) Colima

Mtra. María Guadalupe Alvarado Ibarra.

Universidad de Sonora. México.

MSc. Celso Germán Sánchez Zayas

Universidad de Camagüey, Ignacio Agramonte Loynaz, Cuba

Dra. María Luisa Quintero Soto

Universidad Autónoma del Estado de México

Dr. Eyder Bolivar Mojica

Universidad Católica, Luis Amigó, Medellin, Colombia

Revisores de Textos en Inglés

Mtro. Renato Encinas

Mtra. Cecilia Guadalupe Martínez Solano

\section{Comité científico}

Dr. Rosendo Martínez Jiménez. Universidad Autónoma Benito Juárez de Oaxaca.

Dr. Hugo Neftalí Padilla. Universidad Estatal de Sonora

Dra. María Teresa Gaxiola Sánchez. Universidad de Sonora.

Dr. José Cesar Kaplan. Universidad Estatal de Sonora.

Dr. Alfredo Islas Rodríguez. Universidad de Sonora

Frecuencia de publicación: semestral / 2 números por año y en ocasiones edición especial 
Año 13.

Revista de Investigación

Núm. 34 Edición Especial

Académica sin Frontera

https://revistainvestigacionacademicasinfrontera.unison.mx/index.php/RDIASF

Recibido el 23 de abril de 2020. Dictaminado mediante arbitraje favorablemente 10 de julio de 2020 .

Revista de Investigación Académica sin Frontera (RIASF) con (ISSN: 2007-8870) es un interlocutor internacional de acceso abierto revisado diario en línea en el ámbito del de las Ciencias Económicas Administrativas y Sociales. Su objetivo principal es dar a los trabajos de investigación de calidad. Cubre todas las sub-campos de los campos anteriormente mencionados. Proporciona la plataforma a académicos, estudiantes y profesionales. Sólo pública trabajos de investigación y artículos de revisión inicial. Documento presentado debe cumplir con algunos criterios como, debe ser original, inédita y no estén sometidos a ninguna otra revista.

RIASF es una revista arbitrada / Revisión por pares International. Publicamos documentos sobre una variedad de temas, contextos y estrategias de análisis que examinan la relación entre la rápida evolución para la Sociedad y la tecnología del conocimiento.

REVISTA DE INVESTIGACIÓN ACADÉMICA SIN FRONTERA, Año 13, No.34, Especial tema COVID 19. Julio - diciembre 2020, es una publicación semestral de investigación científica, editada por la Universidad de Sonora, a través de las División de Ciencias Económicas y Sociales, de la Unidad Regional Sur, Blvd. Lázaro Cárdenas No. 100, Col. Francisco Villa, Navojoa, Sonora, Sonora, México, C.P. 85880. Tel. (642) 425-99-54.

https://revistainvestigacionacademicasinfrontera.unison.mx/index.php/RDIASF/,

revistaacademicasinfrontera@unison.mx.

Editor responsable: Francisco Espinoza Morales. Reserva de Derechos al Uso Exclusivo: 042013-121811323700-203 e ISSN: 2007-8870, ambos otorgados por el Instituto Nacional de Derecho de Autor. Inscrita en el Directorio de LATINDEX, con Núm. De folio 20014, folio único 14590. Responsable de la última actualización de este Número, Unidad Informática de la Universidad de Sonora, fecha de la última modificación, 30 de diciembre 2020, indexada a Cite Factor Academic Scientific Journal y Journals Master (IIJIF) y Red Latinoamericana de Revistas Académicas en Ciencias Sociales y Humanidades, (Latín Rev). Las opiniones expresadas por los autores no necesariamente reflejan la postura del editor de la publicación. Se autoriza la reproducción total o parcial de los contenidos e imágenes en la presente publicación siempre y cuando se cuente con la autorización del editor y se cite plenamente la fuente. 
Año 13.

Revista de Investigación

Núm. 34 Edición Especial

Académica sin Frontera

https://revistainvestigacionacademicasinfrontera.unison.mx/index.php/RDIASF

Recibido el 23 de abril de 2020. Dictaminado mediante arbitraje favorablemente 10 de julio de 2020 .

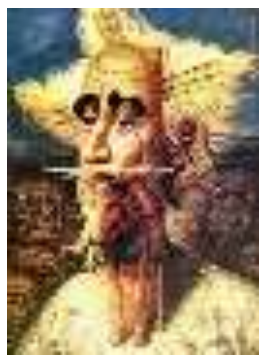

Nos complace anunciar que su diario, "Academic Research Journal Withoutborders" (ISSN/EISSN 2007-8870) fue evaluado positivamente en la indexación Citefactor, ahora la página de la revista está disponible en línea, en caso de cualquier problema.

Journals Master | International Innovative Journal Impact Factor (IIJIF)

Red Latinoamericana de revistas Académicas en Ciencias Sociales y Humanidades
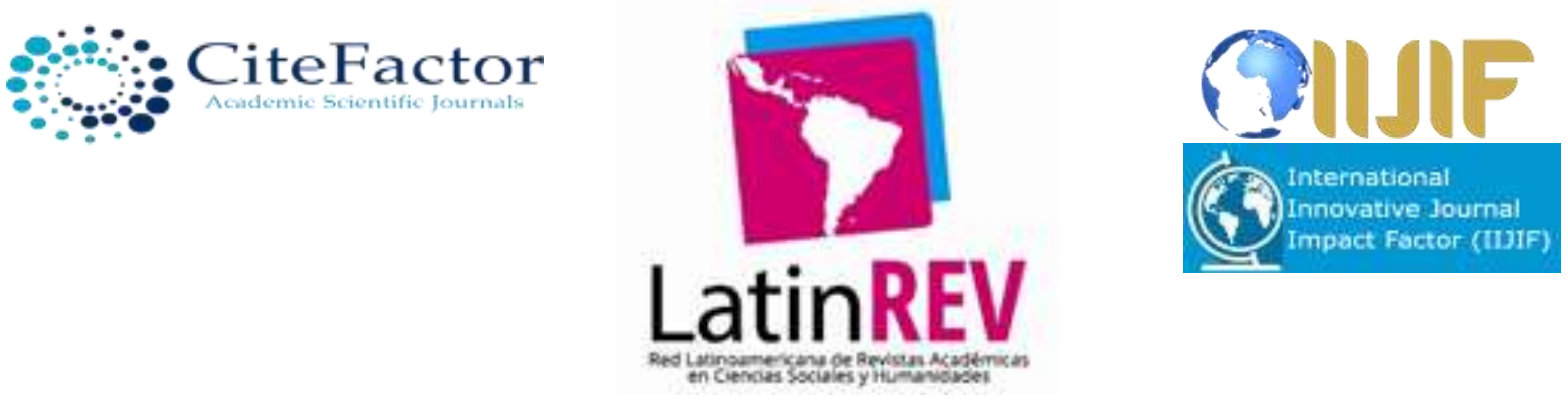

https://www.neliti.com
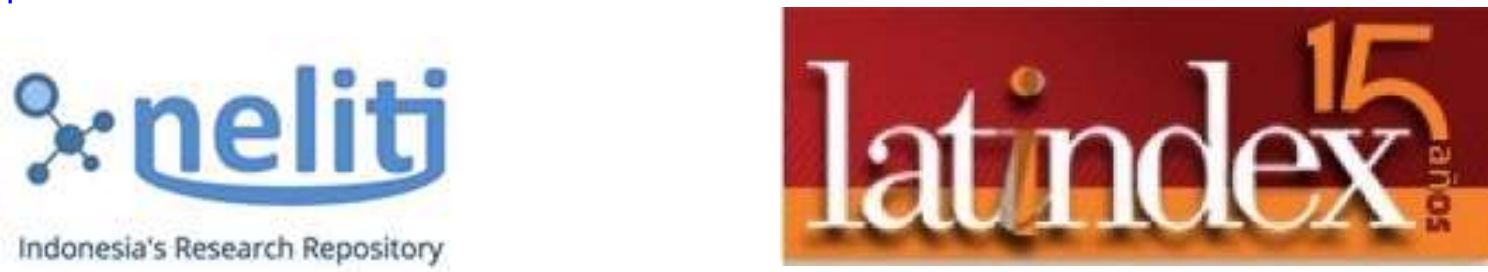